\title{
The Systemic Inflammation on Transradial vs Transfemoral Approach for Coronary Angiography in Patients with Chronic Coronary Syndrome
}

\section{-Does Transradial Coronary Angiography Trigger More Inflammation?}

\author{
Ozge Cetinarslan', Aysem Kaya², Alev Arat Ozkan² \\ ${ }^{1}$ Private Incirli Ethica Hospital, Istanbul, Turkey \\ ${ }^{2}$ Institute of Cardiology, University of Istanbul, Cerrahpasa, Turkey \\ Email: ozgecetinarslan@windowslive.com,kaya34kaya@gmail.com, alevarat@hotmail.com
}

How to cite this paper: Cetinarslan, O., Kaya, A. and Ozkan, A.A. (2021) The Systemic Inflammation on Transradial vs Transfemoral Approach for Coronary Angiography in Patients with Chronic Coronary Syndrome-Does Transradial Coronary Angiography Trigger More Inflammation?. World Journal of Cardiovascular Diseases, 11, 99105 .

https://doi.org/10.4236/wjcd.2021.112011

Received: December 27, 2020

Accepted: February 16, 2021

Published: February 19, 2021

Copyright $\odot 2021$ by author(s) and Scientific Research Publishing Inc. This work is licensed under the Creative Commons Attribution International License (CC BY 4.0).

http://creativecommons.org/licenses/by/4.0/

(c) (i) Open Access

\begin{abstract}
Background: Most of the institutions accept the Transradial Access (TRA) as the first approach for patients undergoing Coronary Angiography (CAG) and Percutaneous Coronary Interventions (PCI). Several studies clearly revealed endothelial injury of coronary arteries triggers inflammatory response. In this study, we aimed to evaluate inflammatory respond to CAG and to compare the inflammatory response of TRA and Transfemoral Access (TFA). Methods: In this single-center prospective study 140 consecutive patients presenting with (Chronical Coronary Syndrome) CCS and underwent transradial or transfemoral CAG between December 2017 and December 2018 were included. After exclusions, left 92 patients were divided into two equal groups as TRA and TFA. The primary endpoints were 2nd hour Tumor Necrosis Factor alpha (TNF $\alpha$ ), $48^{\text {th }}$ hour high-sensitivity C-Reactive Protein (hs-CRP), complication rates, amount of contrast medium, procedure time and fluoroscopy time. Results: Basal characteristics of TRA and TFA groups were similar. A comparison of variables demonstrated that there was no statistical significance in increase in inflammatory markers (TNF $\alpha$, hs-CRP), complication rates, amount of contrast media and procedure time. In contrast, TRA was associated longer fluoroscopy time and higher X-ray dosage, although statistically insignificant. CAG caused a similar increase in hs-CRP in both groups, insignificantly. Conclusion: In contrast to previous studies reporting a higher inflammatory response with TRA, this study demonstrates that inflammatory response caused by CAG is unrelated to access site and similar in
\end{abstract}


TRA and TFA. Longer fluoroscopy time, higher X-ray dosage and longer procedure time in the TRA group indicate the importance of carefully selecting the angiographic route especially in patients with chronic heart failure, chronic renal impairment and malignancy risk. Also, these parameters place importance for physicians own health and woman planning pregnancy.

\section{Keywords}

Transradial Coronary Angiography, Transfemoral Coronary Angiography, Inflammation, TNF $\alpha$, hs-CRP

\section{Introduction}

The first cardiac catheterization procedure was performed by Werner Forssmann in 1929 and selective CAG was described by Mason Sones in the early 1960s [1]. TFA approach which was commonly used, has been compared with radial approach in multiple randomized studies. TRA has been considered as the preferred vascular access site due to vessel-related complications and bleeding related increased morbidity, mortality and hospital stay with TFA, particularly when anticoagulation and antiplatelet therapy are administered [2] [3].

Most of the institutions currently accept the Transradial Access (TRA) as the first choice for patients undergoing CAG and Percutaneous Coronary Interventions (PCI) in regard of data from randomized trials which have shown TRA reduced major bleeding and major adverse cardiac events compared with Transfemoral Access (TFA). TRA has been especially investigating in high-risk population such as women, elderly patients or patients with prior CABG [4] [5] [6]. Studies clearly revealed that CAG and PCI trigger inflammatory response [7] [8] [9]. Higher inflammatory response means poorer outcome. Hs-CRP was assessed due to hs-CRP is gold standard to diagnose inflammation and recent studies have found a certain correlation between hs-CRP and atherosclerosis. TNF $\alpha$ is a powerful proinflammatory cytokine with pleiotropic properties and a key mediator of inflammation. This study is the first head-to-head comparison of inflammation between TRA and TFA in patients presenting with Chronical Coronary Syndrome (CCS) and undergoing CAG without PCI.

\section{Methods}

In this single-center prospective study 140 consecutive patients presenting with CCS and underwent transradial or transfemoral CAG between December 2017 and December 2018 were included. After exclusions, left 92 patients (41 female, 51 male) were divided into two equal groups as TRA group and TFA group. Patients with an estimated glomerular filtration rate (eGFR) of $<30 \mathrm{ml} / \mathrm{min} / 1.7 \mathrm{~m}^{2}$, age $>75$ years, history of CABG, inflammatory diseases or malignancies and those with procedural complications or cross over were excluded. The primary endpoints were $2^{\text {nd }}$ hour TNF $\alpha$ value, $48^{\text {th }}$ hour hs-CRP, complication rates, amount 
of contrast medium, procedure time and fluoroscopy time. Demographic and clinical characteristics, total angiography and fluoroscopy time, contrast media volume, quantity of X-ray, access site, number of puncture attempts, use of sedation, size of sheath and catheters, occurrence of radial artery spasm, contents of intra-arterial cocktails, complications during in hospital period and angiographic findings were recorded. Venous blood samples for hs-CRP and TNF $\alpha$ levels were obtained prior to puncture and post procedure at $2^{\text {nd }}$ and $48^{\text {th }}$ hours. Other biochemical findings (hemogram, blood glucose, creatinine, lipids) as well as patients' height, weight and left ventricular ejection fraction were obtained from hospital records.

All statistical analysis was carried out using SPSS 24.0 software (IBM SPSS for Windows, ver.24). On detection of the sufficiency of sample size, power of all values was described at least $80 \%$ and type 1 error was accepted 5\%. Shapiro-Wilko test $(\mathrm{n}<50)$ was used as test of normality. Continuous variables were presented as mean \pm standard deviation (SD). Categorical variables were presented as percentages. The Independent Samples $t$ test compared the means of two independent groups (TRA vs TFA) in order to determine whether there is statistical evidence that the associated population means are significantly different. The test of nonparametric variables was Mann-Whitney $U$ test. The paired $t$ test and Wilcoxon analysis were used to compare dependent parametric values and dependent nonparametric values, significantly. The correlation values were obtained using the Spearman rank order correlation test. The enumeration data were presented as rate and compared using $\chi^{2}$ test. $\mathrm{P}<0.05$ was considered as statistically significant.

\section{Results}

Mean age of TRA and TFA patients were $57.1 \pm 8.5$ and $57.1 \pm 10.7$ years, respectively. $63.0 \%$ of TRA population and $47.8 \%$ of TFA population were male. To avoid their effects on inflammation, basal characteristics such as hypertension, diabetes mellitus, hyperlipidemia, renal failure, PCI history and smoking of TRA and TFA groups were similar. Basal TNF $\alpha$ and basal hs-CRP values of two groups were insignificant (basal hs-CRP of TRA and TFA patients $3.70 \pm 4.31$ and $4.20 \pm 4.48$, respectively, p: 0.592 . Basal TNF $\alpha$ of TRA and TFA patients 7.10 \pm 3.39 and $6.2 \pm 2.98$, respectively, p: 0.052 ) (Table 1). A comparison of variables demonstrated that there was no significant increase in inflammatory markers (TNF $\alpha$, hs-CRP), complication rates, amount of contrast media, procedure time between two groups (Table 2). On the other hand, TRA was associated longer fluoroscopy time than TFA $(6.01 \pm 3.84$ vs $3.64 \pm 1.55$, p $<0.001)$ and insignificantly higher $\mathrm{X}$-ray dosage.

\section{Discussion}

The comparison of TRA versus TFA with regard to reducing major cardiovascular and cerebrovascular complications was demonstrated with a meta-analysis 
Table 1. Basal characteristics of TRA and TFA groups.

\begin{tabular}{cccccc}
\hline & \multicolumn{2}{c}{ TRA } & \multicolumn{2}{c}{ TFA } & p \\
\cline { 2 - 5 } & $\mathbf{n}$ & $\%$ & $\mathbf{n}$ & $\%$ & \\
\hline Sex (male) & 29 & 63.0 & 22 & 47.8 & 0.142 \\
Hypertension & 33 & 71.7 & 33 & 71.7 & 1.000 \\
Diabetes Mellitus & 22 & 47.8 & 15 & 32.6 & 0.137 \\
Hyperlipidemia & 23 & 50,0 & 18 & 39.1 & 0.294 \\
Renal Failure & 1 & 2.2 & 1 & 2.2 & 1.000 \\
PCI history & 5 & 10.9 & 10 & 21.7 & 0.158 \\
Smoking & 17 & 37.0 & 9 & 19.6 & 0.064 \\
Acetylsalicylic acid & 18 & 39.1 & 17 & 37.0 & 0.830 \\
Clopidogrel & 3 & 6.5 & 6 & 13.0 & 0.292 \\
Ticagrelor & 0 & 0.0 & 1 & 2.2 & 0.315 \\
Statin & 17 & 37.0 & 15 & 32.6 & 0.662 \\
Betablocker & 19 & 41.3 & 16 & 34.8 & 0.519 \\
ACEI/ARB & 22 & 47.8 & 25 & 54.3 & 0.532 \\
DHP-CCB & 6 & 13.0 & 8 & 17.4 & 0.562 \\
Non-DHP-CCB & 1 & 2.2 & 0 & 0.0 & 0.315 \\
\hline
\end{tabular}

PCI: Percutaneous coronary intervention, ACEİARB: Angiotensin converting enzyme inhibitor/ Aldosterone receptor blocker, DHP-CCB: Dihydropyridine calcium channel blocker.

Table 2. Comparison of the primary endpoints.

\begin{tabular}{cccc}
\hline & TRA Mean & TFA Mean & p \\
\hline Hs-CRP $0^{\text {th }}$ hour $(\mathrm{mg} / \mathrm{dl})$ & $3.70 \pm 4.31$ & $4.20 \pm 4.48$ & 0.592 \\
Hs-CRP $48^{\text {th }}$ hour $(\mathrm{mg} / \mathrm{dl})$ & $10.89 \pm 20.34$ & $11.75 \pm 15.64$ & 0.821 \\
Hs-CRP $0^{\text {th }}-48^{\text {th }}$ hour $(\mathrm{mg} / \mathrm{dl})$ & $4.77 \pm 9.73$ & $7.56 \pm 14.04$ & 0.276 \\
TNF $\alpha 0^{\text {th }}$ hour $(\mathrm{pg} / \mathrm{dl})$ & $7.10 \pm 3.39$ & $6.2 \pm 2.98$ & 0.052 \\
TNF $\alpha 2^{\text {nd }}$ hour $(\mathrm{pg} / \mathrm{dl})$ & $6.50 \pm 3.58$ & $5.59 \pm 2.97$ & 0.064 \\
TNF $\alpha 0^{\text {th }}-2^{\text {nd }}$ hour $(\mathrm{pg} / \mathrm{dl})$ & $0.23 \pm 0.70$ & $0.17 \pm 0.37$ & 0.588 \\
X-ray $(\mathrm{Gy})$ & $830.32 \pm 411.79$ & $710.65 \pm 203.27$ & 0.081 \\
Amount of contrast media $(\mathrm{ml})$ & $70.22 \pm 22.58$ & $62.07 \pm 20.04$ & 0.070 \\
Procedure time $(\mathrm{min})$ & $12.30 \pm 6.62$ & $10.30 \pm 3.62$ & 0.076 \\
Flouroscopy time $(\mathrm{min})$ & $6.01 \pm 3.84$ & $3.64 \pm 1.55$ & $<0.001$ \\
\hline
\end{tabular}

TRA: Transradial access, TFA: transfemoral access, hs-CRP: high-sensitivity C-reactive protein, hs-CRP 0 : Basal high-sensitivity C-reactive protein, hs-CRP $48: 48^{\text {th }}$ hour high-sensitivity C-reactive protein, hs-CRP 0 - 48: Difference between basal and $48^{\text {th }}$ hour high-sensitivity C-reactive protein between, TNF $\alpha$ : tumor necrosis factor alpha, TNF $\alpha$ 0: Basal tumor necrosis factor alpha, TNF $\alpha$ 2: Second hour tumor necrosis factor alpha, TNF $\alpha 0$ - 2: Difference between basal and $2^{\text {nd }}$ hour tumor necrosis factor alpha, Gy: Gray

including four important trials called as MATRIX-ACCESS, RIVAL, SAFE-PCI and STEMI-RADIAL [10]. According to meta-analyses, TRA reduced death from 
all causes during the first 30 days following intervention, bleeding and local complications at the access site. In addition, TRA was associated with a reduction in length of hospital stay and more of the patients preferred the TRA for their next procedure [11]. In patients with CCS, inflammation has been reported to be correlated with plaque progression. Even low-grade inflammation that is defined by high plasma levels of hs-CRP and TNF $\alpha$ was described a significant risk factor for coronary spasm and coronary artery disease (CAD) [12] [13]. Only rare studies are available about inflammatory response of patients who experienced CAG without PCI and our study is the first head-to-head comparison of inflammation between TRA and TFA in patients presenting with CCS and undergoing CAG without PCI.

In contrast to previous single arm studies reporting a higher inflammatory response with TRA, this study demonstrates that inflammatory response caused by CAG is unrelated to access site. This result can be related to exclusion of multiple punctures via experienced operators in TRA population. Longer fluoroscopy time, higher X-ray dosage and longer procedure time in the TRA group indicate the importance of carefully selecting the angiographic route especially in patients with chronic heart failure, chronic renal impairment and malignancy risk. Also, these parameters place importance for physicians own health and woman planning pregnancy.

\section{Conclusion}

In contrast to previous studies reporting a higher inflammatory response with TRA, this study demonstrates that inflammatory response caused by CAG is unrelated to access site and similar in TRA and TFA. Longer fluoroscopy time, higher X-ray dosage and longer procedure time in the TRA group indicate the importance of carefully selecting the angiographic route especially in patients with chronic heart failure, chronic renal impairment and malignancy risk. Also, these parameters place importance for physicians own health and woman planning pregnancy.

\section{Limitations}

Firstly, this was a single center study with a limited number of patients. Most quantitative analyses require a fairly large sample. Secondly, only the TNF $\alpha$ and hs-CRP have been assessed due to hs-CRP is gold standard to diagnose inflammation. Finally, patients with eGFR of $<30 \mathrm{ml} / \mathrm{min} / 1.7 \mathrm{~m}^{2}$, age $>75$ years, history of CABG, inflammatory diseases or malignancies and those with procedural complications or cross over were not included in the study group. Including these fragile patients can provide more generalizable information.

\section{Funding}

The research was supported by University of Istanbul, Cerrahpasa, Scientific Researches Department with project number of 31333. 


\section{Conflicts of Interest}

The authors declare no conflicts of interest regarding the publication of this paper.

\section{References}

[1] Soydan, E. and Akın, M. (2018) Coronary Angiography Using the Left Distal Radial Approach-An Alternative Site to Conventional Radial Coronary Angiography. The Anatolian Journal of Cardiology, 19, 243-248. https://doi.org/10.14744/AnatolJCardiol.2018.59932

[2] Brueck, M., Bandorski, D., Kramer, W., Wieczorek, M., Höltgen, R. and Tillmanns, H. (2009) A Randomized Comparison of Transradial versus Transfemoral Approach for Coronary Angiography and Angioplasty. JACC: Cardiovascular Interventions, 2, 1047-1054. https://doi.org/10.1016/j.jcin.2009.07.016

[3] Cantor, W.J., Puley, G., Natarajan, M.K., Dzavik, V., Madan, M., Fry, A., et al. (2005) Radial versus Femoral Access for Emergent Percutaneous Coronary Intervention with Adjunct Glycoprotein IIb/IIIa Inhibition in Acute Myocardial Infarction-The RADIAL-AMI Pilot Randomized Trial. American Heart Journal, 150, 543-549. https://doi.org/10.1016/j.ahj.2004.10.043

[4] Amro, A., Mansoor, K., Amro, M., et al. (2020) Transradial versus Transfemoral Approach for Coronary Angiography in Females with Prior Bypass Surgery. Cureus, 12, e6797. https://doi.org/10.7759/cureus.6797

[5] Kedev, S., Sukmawan, R., Kalpak, O., et al. (2016) Transradial versus Transfemoral Access for Female Patients Who Underwent Primary PCI in STEMI: Two Years Follow-Up Data from Acute STEMI Interventional Registry. International Journal of Cardiology, 217, 16-20. https://doi.org/10.1016/j.ijcard.2016.06.222

[6] He, P.-Y., Yang, Y.-J., Qiao, S.-B., et al. (2015) A Comparison of the Transradial and Transfemoral Approaches for the Angiography and Intervention in Patients with a History of Coronary Artery Bypass Surgery: In-Hospital and 1-Year Follow-Up Results. Chinese Medical Journal, 128, 762-767. https://doi.org/10.4103/0366-6999.152488

[7] Goldberger, A., Zinder, O., Zdorovyak, A., et al. (2003) Diagnostic Coronary Angiography Induces a Systemic Inflammatory Response in Patients with Stable Angina. American Heart Journal, 146, 819-823. https://doi.org/10.1016/S0002-8703(03)00407-1

[8] Farb, A., Sangiorgi, G., Carter, A.J., et al. (1999) Pathology of Acute and Chronic Coronary Stenting in Humans. Circulation, 99, 44-52. https://doi.org/10.1161/01.CIR.99.1.44

[9] Rogers, C., Welt, F.G., Karnovsky, M.J. and Edelman, E.R. (1996) Monocyte Recruitment and Neointimal Hyperplasia in Rabbits. Coupled Inhibitory Effects of Heparin. Arteriosclerosis, Thrombosis, and Vascular Biology, 16, 1312-1318. https://doi.org/10.1161/01.ATV.16.10.1312

[10] Halabi, A.S., Burke, L., Hussain, F., Lopez, J., Mathew, V., Bernat, I. and Shroff, A. (2019) Radial versus Femoral Approach in Woman Undergoing Coronary Angiograph: A Meta-Analysis of Randomized Controlled Trials. Journal of Invasive Cardiology, 318, 335-340.

[11] Kolkailah, A.A., Alreshq, R.S., Muhammed, A.M., Zahran, M.E., Anas El-Wegoud, M. and Nabhan, A.F. (2018) Transradial versus Transfemoral Approach for Diagnostic Coronary Angiography and Percutaneous Coronary Intervention in People 
with Coronary Artery Disease. Cochrane Database of Systematic Reviews, Issue 4, Article No. CD012318. https://doi.org/10.1002/14651858.CD012318.pub2

[12] Hung, M.J., Cherng, W.J., Yang, N.I., Cheng, C.W. and Li, L. (2005) Relation of High-Sensitivity C-Reactive Protein Level with Coronary Vasospastic Angina Pectoris in Patients without Hemodynamically Significant Coronary Artery Disease. The American Journal of Cardiology, 96, 1484-1490.

https://doi.org/10.1016/j.amjcard.2005.07.055

[13] Itoh, T., Mizuno, Y., Harada, E., Yoshimura, M., Ogawa, H. and Yasue, H. (2007) Coronary Spasm Is Associated with Chronic Low-Grade Inflammation. Circulation Journal, 71, 1074-1078. 